\title{
Psychological and demographic factors associated with fatigue and social adjustment in young people with severe chronic fatigue syndrome/myalgic encephalomyelitis: a preliminary mixed- methods study
}

\author{
Sheila Ali ${ }^{1} \cdot$ Lucy Adamczyk $^{2} \cdot$ Mary Burgess $^{1} \cdot$ Trudie Chalder $^{3}$ CD
}

Received: February 22, 2018/Accepted: January 5, 2019/Published online: January 25, 2019

(C) The Author(s) 2019

\begin{abstract}
This mixed-methods study investigated factors associated with fatigue, disability and school attendance in young people with severe CFS/ME. Participants' illness experiences were also explored. Questionnaires were completed at baseline (T1) and approximately 5 months later (T2). There were 51 participants aged between 12 and 25 , with a mean age of 18.8 years (SD 3.4). At T1, participants reported severe fatigue and poor social adjustment. Stronger fear avoidance beliefs at T1 were associated with higher fatigue at $\mathrm{T} 2$, and with worse social adjustment at T1 and T2. Female gender was associated with lower work/school attendance at $\mathrm{T} 1$ and $\mathrm{T} 2$ but not with higher fatigue or worse social adjustment. Having accessed treatment was associated with reporting lower levels of work/school attendance at T1 and T2. Multivariate analyses of key outcomes identified significant associations between stronger fear avoidance beliefs and worse social adjustment at $\mathrm{T} 2$, and between female gender and lower work/school attendance at T2. It was clear from the qualitative data that severe CFS/ME negatively impacted on many aspects of young people's lives. Fearful beliefs about
\end{abstract}

Electronic supplementary material The online version of this article (https://doi.org/10.1007/s10865-019-00010-x) contains supplementary material, which is available to authorized users.

Trudie Chalder

trudie.chalder@kcl.ac.uk

1 South London and Maudsley NHS Foundation Trust, London, UK

2 Institute of Psychiatry, Psychology and Neuroscience, King's College London, London, UK

3 Department of Psychological Medicine, Institute of Psychiatry, Psychology and Neuroscience, King's College London, London, UK activity could be targeted using cognitive-behavioural interventions.

Keywords Chronic fatigue syndrome Adolescent health . Disability · Fatigue

\section{Introduction}

Chronic fatigue syndrome (CFS), also known as myalgic encephalomyelitis/encephalopathy (ME), is characterised by enduring and disabling fatigue. It is usually accompanied by persistent symptoms such as muscle pain, cognitive dysfunction and sleep disturbance (Fukuda et al., 1994; Sharpe et al., 1991). In adolescents, the estimated prevalence of CFS/ME is 0.1-1.29\% (Chalder et al., 2003; Crawley et al., 2011; Farmer et al., 2004; Rimes et al., 2007). Due to a lack of research, prevalence rates for severe CFS/ME have not yet been established (Strassheim et al., 2017).

There is no universally accepted diagnostic test for CFS/ $\mathrm{ME}$ and the diagnosis is difficult to operationalise due to the heterogeneity of the illness. There is a wide range of diagnostic criteria including the Oxford criteria, CDC criteria, London criteria and NICE criteria (Fukuda et al., 1994; Sharpe et al., 1991; The National Task Force on Chronic Fatigue Syndrome, 1994; National Institute for Health and Clinical Excellence, 2007). Although there is no consensus on the most acceptable criteria, the CDC and Oxford criteria are the most widely used (Brurberg et al., 2014; Fukuda et al., 1994, Sharpe et al. 1991), the Oxford criteria being more inclusive.

The symptoms of CFS/ME can have unwanted impacts upon several aspects of everyday life. Young people with CFS/ME may find themselves missing school, having dif- 
ficulties maintaining friendships, and/or dealing with strained family relationships (Burgess \& Chalder, 2011; Parslow et al., 2017; Rangel et al., 2000; Sankey et al., 2006; Taylor et al., 2016). At its most severe, CFS/ME in young people can result in extremely high levels of disability, which can leave the young person bed-bound or unable to attend school (Rangel et al., 2000). Many may need to use a wheelchair to mobilise, and others may be too unwell to leave their homes.

Research evidence on the trajectory and prognosis of CFS/ME in young people is inconclusive (Gill et al., 2004; Norris et al., 2017; Rangel et al., 2000; Rimes et al., 2007; Sankey et al., 2006). However it has been suggested that CFS/ME should be treated early in order for patients to receive the best outcome (Burgess \& Chalder, 2011; Sankey et al., 2006).

Some young people with CFS/ME have a co-morbid diagnosis. Knight et al. (2013) found that $51 \%$ of children and adolescents attending a specialist service reported having a comorbid disorder. Moreover, in a registry-linkage study of children and adolescents within the general population in Norway, Bakken et al. (2016) found that a group with CFS were more likely to report a comorbid diagnosis than a group with type 1 diabetes, and a group from the general population. Comorbidities included anxiety, depression, diabetes, asthma and migraine. This fits with the growing body of evidence that demonstrates a clear relationship between anxiety or depression and CFS in children and adolescents (Bould et al., 2013; Crawley et al., 2009; Loades et al., 2016; Stoll et al., 2017). It is important to note that some comorbidities such as migraine may be associated with psychological factors such as depression (Arita et al., 2013).

The aetiology of CFS/ME is uncertain (Richards, 2000). However a stress-diathesis model of CFS has been proposed. This posits that some individuals may have a greater risk or vulnerability to stress. This may be influenced by a range of different factors such as genes, psychiatric disorder, personality, self-esteem, adverse life events, and parental factors. The onset of CFS may be triggered by a viral infection, illness, or stressful event. Fatigue and CFS symptoms may subsequently be maintained long-term by perpetuating factors such as fearful beliefs about symptoms, excessive resting, and avoidance behaviour (Lievesley et al., 2014).

In line with this model, a number of risk factors have been identified which are associated with persistent fatigue, chronic disabling fatigue, and CFS in adolescents and young people. These include female gender, older age, higher IQ, genetic predisposition, viral infection, comorbid anxiety or depression, maternal distress, family adversity, and physical activity level (Collin et al., 2016, 2018a; Hickie et al., 1999; Katz et al., 2009; Rimes et al., 2007).
Cognitive factors such as symptom focusing and a preference for resting can predict poorer outcome once the symptoms have developed (Gray \& Rutter, 2007; Richards et al., 2005). In a case control study nested within a cohort, fear avoidance beliefs, such as "I am afraid that I will make my symptoms worse if I exercise" correlated with physical functioning and may well have arisen from having had experiences of worsening fatigue after engaging in activity (Loades et al., in submission).

There is a dearth of existing research investigating factors associated with fatigue or social adjustment in young people with severe CFS/ME. One study by Rangel et al. (2000) found that young people with severe CFS were more likely to recover if they had a higher socioeconomic status, if they had a physical trigger for their illness(e.g. flu), and if the onset of their illness was in the autumn school term. To our knowledge, there are no other studies which have investigated predictors of outcome in young people with severe CFS. There is also an absence of qualitative or descriptive work on the lived experience of young people with severe CFS/ME. This study therefore had two key aims: (1) to investigate whether factors such as fear avoidance, gender, age, and access to treatment were associated with the key outcomes of fatigue, social adjustment and work/school attendance in young people with severe CFS/ME; and (2) to explore the lived experience of young people with severe CFS/ME. This was a mixed-methods cohort study which involved the collection and analysis of qualitative and quantitative data. Data were collected at baseline (T1) and several months later (T2). It was expected that stronger fear avoidance beliefs about symptoms at T1 would be associated with more severe fatigue, as well as poorer social adjustment and lower work/school attendance at T2. It was also expected that access to treatment would be associated with higher work/ school attendance at T2. Additional analyses were also conducted in order to adjust for the time between completion of the $\mathrm{T} 1$ and $\mathrm{T} 2$ questionnaires.

\section{Methods}

\section{Design}

This study received ethical approval from the Psychiatry, Nursing and Midwifery sub-committee of King's College London Research Ethics Committee (reference PNM/12/ 13-141). Data collection took place between July 2014 and May 2015. This study employed a short-term prospective design. Questionnaire data were collected in two waves: at baseline (T1), and at follow-up (T2), which was 3-9 months later. The average time between questionnaires was 4.91 months (SD 1.32). All questionnaire measures 
were collected at both $\mathrm{T} 1$ and $\mathrm{T} 2$. Written informed consent was obtained from study participants, with additional parental consent for participants under 18 .

\section{Recruitment}

Participants were recruited from a charity for young people with CFS/ME. This setting was chosen because the charity had contact with a relatively large number of young people who were severely affected by CFS/ME. Recruitment took part in two phases. In the first phase, invitation letters were sent to members of the charity who had severe CFS/ME. The second phase of recruitment involved extending the study invitation to other members of the charity. Advertisements were placed on the charity's mailing lists and social networking websites. Individuals who expressed an interest in taking part were asked to contact a named person at the charity, at which point they completed a screening questionnaire to check whether they met the eligibility criteria for the study.

To be eligible for inclusion in the study, participants were required to have a self-reported diagnosis of CFS/ME, and to be fluent in English (this is because the questionnaire measures have been validated in English, and there were no resources available for translation of these measures into other languages). Participants also had to be aged between 11 and 26 (the study originally sought to recruit young people aged 11-18 but the upper age limit was raised to 26 in order to increase recruitment rates, and because members of the charity could be aged between 11 and 26). An additional requirement was that participants had severe CFS. This was assessed using the functional ability scale (Moss, 2005). This self-report rating scale describes levels of functioning in young people with CFS/ ME. Possible scores range from 0 (very severely affected) to $100 \%$ (fully recovered), with lower scores indicating poorer functioning and more severe CFS/ME. In phase 1 of recruitment, a score of $\leq 30 \%$ was required for inclusion in the study. An individual functioning at the $30 \%$ level would experience moderate to severe symptoms at rest, and severe symptoms following any activity. They would spend most of the day resting.

In phase 2 of recruitment, in order to increase recruitment rates, the threshold for severity was raised to $50 \%$. At this level, a person would still experience moderate symptoms which would increase following activity. Three participants were recruited during this second phase.

\section{Questionnaires}

Participants were asked to complete a questionnaire which consisted of open-ended and closed questions. This was piloted with a young person with CFS/ME and their par- ents, and adjusted according to their feedback. To ensure that the study was accessible to participants with high levels of disability and to reduce the potential burden, participants were advised that they could ask for help from their parent with completing the questionnaire and that they could take breaks. Questionnaires were sent by mail. Reminders were sent to non-responders. Participants were asked a number of questions related to demographic characteristics and were also asked to complete some validated questionnaires.

\section{Participant characteristics}

Participants were asked questions about demographic characteristics such as age and gender. They were also asked questions relating to their medical history, for example whether they had been diagnosed with CFS/ME and by whom, whether they had a family member with CFS/ME, and whether they had any comorbid problems. In order to ascertain whether they met the Oxford criteria for CFS, participants were also asked about the duration, chronicity and severity of their fatigue (Sharpe et al., 1991). We used the broader Oxford criteria to minimise the likelihood of excluding people with CFS.

Mobility and access to treatment: participants were asked about their current level of mobility, for example whether they were bed-bound, and whether they were using a wheelchair or any assistive equipment (and if so, what type). There was also a series of questions about treatment. For example, participants were asked whether they were currently accessing treatment or had previously accessed it. This was coded as a dichotomous (yes/no) variable for the analysis. They were also asked to specify the type of treatment they were accessing including whether they were taking medication.

Meaningful education/work: participants were asked if they had been able to attend school/college/university/work in the past year, and if they answered in the affirmative, they were asked to state the number of days per week they had been attending. This was converted into a percentage, which was used as an outcome variable in the analysis.

\section{Questionnaires}

Participants were also asked to complete the following questionnaires:

\section{Fatigue}

Fatigue severity was measured using the Chalder fatigue scale, which is a reliable and valid measure of physical and mental fatigue (Cella \& Chalder, 2010; Chalder et al., 1993). It has 11 items that sum to a maximum possible total 
score of 33. Participants were also asked to complete a visual analogue scale rating their fatigue severity on a scale of $1-100$.

\section{Social adjustment}

The Work and Social Adjustment scale (Cella et al., 2011; Mundt et al., 2002) is a five-item scale measuring social adjustment and participation in life. Each item receives a score out of 8 . The five items are summed to obtain a total score out of a maximum of 40, with higher scores indicating worse social adjustment. The scale is primarily used with adults but was adapted for young people by adding 'school' and 'college' to the 'work' item.

\section{Fear avoidance beliefs}

This study used the fear avoidance subscale of the Cognitive Behavioural Responses questionnaire (Ryan et al., 2017; Skerrett \& Moss-Morris, 2006; Stahl et al., 2014). The items measure fearful beliefs about activity, and beliefs relating to the avoidance of activity. The subscale consists of six items, each of them scored from 0 to 4 . Scores were calculated out of a total of 24 , with higher scores indicating stronger fearful beliefs about activity.

\section{Presence of anxiety or depression}

The presence of anxiety or depression was screened for using the PHQ-2 and the GAD-2 (Arroll, 2003; Kroenke et al., 2007). Each of these scales has 2 items that are scored from 0 to 3 and therefore the total score for both scales can range from 0 to 6 , with higher scores indicating more severe depression or anxiety. A score of 3 or more for each scale indicates the presence of anxiety or depression respectively.

\section{Open-ended question}

An open-ended question was included which asked all participants to elaborate on their experiences of being a member of the charity and having CFS/ME, including anything that affected their life in a positive or negative way. They were also asked to give feedback about the charity.

\section{Data analysis}

Data analyses were conducted within version 23 of IBM SPSS Statistics (IBM Corp., 2015). Demographic characteristics were described using frequencies and measures of central tendency, as appropriate. Distributions were checked using Q-Q plots. Relationships between individual variables at T1 and T2 were examined using correlations. Point-biserial correlations were used for correlations using one categorical variable and one continuous variable. The SPSS bootstrapping procedure was used with 1000 bootstrap samples, and bias-corrected 95\% CI were calculated.

Subsequently, multiple regression analyses were conducted to examine whether factors (such as demographic characteristics and fear avoidance beliefs) were associated with fatigue, social adjustment and work/school attendance at T2.

The responses to the open-ended text questions were analysed using conventional content analysis (Hsieh \& Shannon, 2005). The process involved inductive coding of qualitative data into categories: fragments of text were coded at the smallest meaningful unit, and where possible, text fragments were coded into mutually-exclusive categories (Sommer, 2006). The coder recorded the frequency of occurrence of each category, and then grouped similar categories together into over-arching themes.

\section{Results}

Invitation letters were sent to 390 young people (and parents of those aged under 18). In a second phase of recruitment, three further participants were recruited via advertisements on the charity's social media pages and email lists. In total, 68 consent forms and 56 questionnaires were returned.

Of the 56 participants who returned questionnaires and consent forms, 2 were excluded because they had recovered. Two participants were excluded due to invalid consent forms. One participant was excluded because the questionnaire was completed entirely by the participant's parent without the involvement of the young person themselves.

51 young people were included in the analysis. All completed questionnaires at baseline (T1). Thirty-eight (74.5\%) participants responded at the follow-up stage (T2).

\section{Baseline characteristics}

Demographic characteristics of participants are shown in Table 1. Participants were aged between 12 and 25, with a mean age of 18.8 years (SD 3.4). Participants reported a mean illness duration of 5.7 years (SD 4.0).

All participants reported that they had a diagnosis of CFS/ME and 43 participants $(84.3 \%)$ met the Oxford criteria for CFS/ME (Sharpe et al., 1991). All participants except one stated that their diagnosis had been given or 
Table 1 Baseline sample characteristics

\begin{tabular}{lr}
\hline Variable & $n(\%)$ \\
\hline Gender & $7(13.7)$ \\
Male & $44(86.3)$ \\
Female & \\
Age group & $18(35.3)$ \\
$11-17$ & $33(64.7)$ \\
$18-26$ & \\
Family member with CFS/ME & $6(11.8)$ \\
Yes & $44(86.3)$ \\
No & $1(2)$ \\
Not stated & \\
Type of treatment being accessed & $6(11.8)$ \\
CBT, GET or both & $19(37.3)$ \\
CBT, GET or both with at least one other treatment & $2(3.9)$ \\
Occupational therapy & $2(3.9)$ \\
Occupational therapy with other treatment & $2(3.9)$ \\
Complementary therapies & $2(3.9)$ \\
Complementary therapies with other treatment & $2(3.9)$ \\
Complementary therapies and Occupational Therapy & $6(11.8)$ \\
Other & $10(19.6)$ \\
Not stated & \\
\hline
\end{tabular}

CFS/ME Chronic fatigue syndrome/myalgic encephalomyelitis (or encephalopathy), $C B T$ cognitive behaviour therapy, GET graded exercise therapy

confirmed by their GP, a consultant, specialist doctor, or physiotherapist.

Thirty-seven $(72.5 \%)$ participants reported using assistive equipment such as crutches, walking frames, ramps, stair-lifts and shower chairs. Thirty-three participants $(64.7 \%)$ reported that they used a wheelchair. Nine participants $(17.6 \%)$ reported that they were bed-bound. Fortyfour participants $(86.3 \%)$ stated that they were taking medication.

Almost two-thirds of the participants reported having a comorbid illness in addition to CFS/ME (58.8\%). Examples included fibromyalgia, depression, anxiety, and obsessive compulsive disorder (OCD). Forty-one participants $(80.4 \%)$ stated that they had accessed at least one form of treatment. As seen in Table 1, patients had accessed a variety of treatments including CBT (cognitive behaviour therapy) and graded exercise therapy (GET) as well as occupational therapy and complementary therapies.

Fourteen participants $(27.5 \%)$ had been able to attend school, college or work in the past year. Ten participants (19.6\%) stated that they had a home tutor. Nine participants (17.6\%) had accessed online learning.

As can be seen in Table 2, at T1, participants reported mean scores on the social adjustment scale which surpassed the clinical cut-off threshold of 20 and indicated a moderately severe or worse level of psychopathology (Mundt et al., 2002). They also reported a mean fatigue score which surpassed the cut-off score of 19 out of 33 reported by Norris et al. (2017) for chronic disabling fatigue (a proxy for clinician-diagnosed chronic fatigue syndrome). Similarly, participants also reported high levels of fatigue on the fatigue visual analogue scale. At T1, 16 participants (31.4\%) scored above 3, the cut-off threshold for anxiety, and $16(31.4 \%)$ scored above the cut-off threshold for depression. T2 scores were not considerably different from $\mathrm{T} 1$ scores, and with the exception of anxiety and depression, still exceeded cut-off thresholds for impairment/severity.

\section{Correlations}

The results of the correlation analyses can be seen in Table 3. In summary, significant associations were found between the following variables: stronger fear avoidance beliefs were associated with higher levels of fatigue at $\mathrm{T} 1$, and with worse social adjustment at T1 and T2. Female gender was significantly associated with lower levels of school attendance at T1 and T2. Similarly, access to treatment was associated with lower levels of school attendance at $\mathrm{T} 1$ and $\mathrm{T} 2$.

\section{Multiple regression analyses}

Multiple regression analyses showed that a model containing age, gender and fear avoidance beliefs at $\mathrm{T} 1$ pre-

Table 2 Scores for key outcomes at T1 and T2

\begin{tabular}{lll}
\hline Score & $\begin{array}{l}\text { T1 } \\
\text { Mean (SD) }\end{array}$ & Mean(SD) \\
\hline Fatigue (out of a maximum of 33) & $25.85(6.19)$ & $26.21(6.67)$ \\
Fatigue visual analogue scale (out of a maximum of 100) & $76.81(20.74)$ & $73.77(24.30)$ \\
Social adjustment scale (out of a maximum of 40) & $31.18(7.97)$ & $30.68(9.35)$ \\
Percentage work/school attendance (out of a maximum of 100\%) & $14.37(28.70)$ & $20.53(32.37)$ \\
Depression (out of a maximum of 6) & $2.44(2.14)$ & $2.53(1.90)$ \\
Anxiety (out of a maximum of 6) & $2.36(2.15)$ & $2.50(2.02)$ \\
\hline
\end{tabular}


Table 3 Correlation analyses examining relationships between independent variables and key outcomes at T1 and T2

\begin{tabular}{|c|c|c|c|c|c|c|}
\hline & $\begin{array}{l}\text { T1 social } \\
\text { adjustment }\end{array}$ & $\begin{array}{l}\mathrm{T} 2 \text { social } \\
\text { adjustment }\end{array}$ & T1 fatigue & $\mathrm{T} 2$ fatigue & $\begin{array}{l}\text { T1 work/school } \\
\text { attendance }\end{array}$ & $\begin{array}{l}\text { T2 work/school } \\
\text { attendance }\end{array}$ \\
\hline $\begin{array}{l}\text { T1 fear } \\
\text { avoidance }\end{array}$ & $.51 * * *(.24 . .69)$ & $.34 *(-.02, .60)$ & $.47 * * *(.21, .69)$ & $.07(-.25, .44)$ & $-.20(-.47, .09)$ & $-.16(-.46, .19)$ \\
\hline Gender & $.21(-.10, .54)$ & $.26(-.05, .58)$ & $.14(-.14, .39)$ & $.21(-.08, .55)$ & $-.52 * * *(-.84,-.07)$ & $-.43 * *(-.75, .07)$ \\
\hline $\begin{array}{l}\text { Access to } \\
\text { treatment }\end{array}$ & $.19(-.12, .49)$ & $.20(-.18, .62)$ & $-.17(-.39, .13)$ & $-.04(-.25, .25)$ & $-.37 * *(-.71, .12)$ & $-.42 * *(-.75, .12)$ \\
\hline
\end{tabular}

Data shown are correlation co-efficients(95\% CI upper and lower limits)

$* p<.05$

$* * p<.01$

$* * * p<.001$

Table 4 Multivariate models examining factors associated with social adjustment, work/school attendance, and fatigue at T2

\begin{tabular}{|c|c|c|c|c|c|}
\hline & $b$ & $S E b$ & $\beta$ & $t$ & $p$ \\
\hline \multicolumn{6}{|c|}{ Model 1 (Outcome: social adjustment at T2) } \\
\hline Age & .50 & .42 & .19 & 1.19 & .121 \\
\hline Gender & 7.02 & 3.88 & .28 & 1.81 & .040 \\
\hline Fear avoidance & .72 & .34 & .33 & 2.11 & .022 \\
\hline \multicolumn{6}{|c|}{ Model 2 (Outcome: work/school attendance at T2) } \\
\hline Age & -1.97 & 1.55 & -.21 & -1.27 & .107 \\
\hline Gender & -43.23 & 15.88 & -.45 & -2.72 & .006 \\
\hline Fear avoidance & -1.31 & 1.24 & -.17 & -1.05 & .151 \\
\hline \multicolumn{6}{|c|}{ Model 3 (Outcome fatigue at T2) } \\
\hline Age & -.061 & .34 & -.03 & -.183 & .428 \\
\hline Gender & 3.86 & 3.08 & .21 & 1.26 & .109 \\
\hline Fear avoidance & .13 & .27 & .08 & .47 & .320 \\
\hline
\end{tabular}

dicted social adjustment at $\mathrm{T} 2\left(\mathrm{R}^{2}=.22, F(3,33)=3.16\right.$, $p=.04)$. As can be seen in Table 4 , stronger fear avoidance beliefs at $\mathrm{T} 1$ were specifically associated with poorer social adjustment at T2. Female gender was also associated with poorer social adjustment at $\mathrm{T} 2$.

Secondly, a model containing age, gender and fear avoidance beliefs at $\mathrm{T} 1$ predicted work/school attendance at $\mathrm{T} 2\left(\mathrm{R}^{2}=.26, F(3,28)=3.22, p=.04\right)$. Specifically, female gender was associated with lower work/school attendance at $\mathrm{T} 2$.

Finally, a model containing fear avoidance beliefs, age and gender was not predictive of fatigue at $\mathrm{T} 2\left(\mathrm{R}^{2}=.05\right.$, $F(3,33)=.59, p=.63)$.

\section{Adjustment for time}

As seen in the Table A1 in the supplementary material, the addition of time as a covariate did not alter the findings of the multiple regression analyses except that the variables fear avoidance and gender were not significant predictors of T2 social adjustment.

\section{Access to treatment}

A model containing age, gender and access to treatment did not significantly predict social adjustment: $\mathrm{R}^{2}=.14$, $F(3,32)=1.70, p=.19$. On the other hand, a model containing age, gender and access to treatment significantly predicted work/school attendance: $\mathrm{R}^{2}=.26$, $F(3,28)=3.36, p=.03$. However as can be seen in Table 5, none of the individual variables were significantly associated with outcome. Finally, a model containing age, gender and access to treatment was not predictive of $\mathrm{T} 2$ fatigue: $\mathrm{R}^{2}=.11, F(3,32)=1.28, p=.30$. However gender was significantly associated with outcome.

\section{Qualitative analysis}

The content analysis revealed six over-arching themes which are shown in Table 6 and summarised briefly below. 
Table 5 Multiple regression models investigating the impact of access to treatment, age and gender on key outcomes

\begin{tabular}{|c|c|c|c|c|c|}
\hline & $b$ & $S E b$ & $\beta$ & $t$ & $p$ \\
\hline \multicolumn{6}{|c|}{ Model 1 (Outcome: social adjustment at T2) } \\
\hline Age & .51 & .46 & .18 & 1.10 & .141 \\
\hline Gender & 7.73 & 5.29 & .29 & 1.46 & .077 \\
\hline Access to treatment & 1.25 & 5.27 & .05 & .24 & .408 \\
\hline \multicolumn{6}{|c|}{ Model 2 (Outcome: work/school attendance at T2) } \\
\hline Age & -1.97 & 1.54 & -.21 & -1.28 & .106 \\
\hline Gender & -27.82 & 20.08 & -.29 & -1.39 & .089 \\
\hline Access to treatment & -21.75 & 18.26 & -.25 & -1.19 & .12 \\
\hline \multicolumn{6}{|c|}{ Model 3 (Outcome fatigue at T2) } \\
\hline Age & -.15 & .34 & -.08 & -.45 & .328 \\
\hline Gender & 7.49 & 3.89 & .38 & 1.93 & .032 \\
\hline Access to treatment & -4.82 & 3.88 & -.25 & -1.24 & .112 \\
\hline
\end{tabular}

\section{Theme 1: positive factors and sources of support}

Nearly a third of the categories were related to positive factors and sources of social support. The responses revealed that participants were grateful for the charity's help in advocating for young people with CFS/ME, raising awareness, and providing the participants with social support networks where they could interact with other young people with CFS/ME. One participant commented: 'It is so reassuring that ME sufferers are not alone'.

\section{Theme 2: link between CFS/ME symptoms and other variables}

Just under $10 \%$ of the categories were in relation to participant's views about possible links between CFS/ME symptoms and other variables. For example, one participant suggested that low mood is a consequence of having CFS/ME: 'People who feel/sad low etc. is a side effect of ME so should not be considered causational to ME. Anybody would feel low/sad if their ability to live has been impaired'.

\section{Theme 3: the lived experience of having CFS/ME}

Participants' responses illustrated the reality of living with CFS/ME, and it was apparent from the comments that symptoms of CFS/ME and levels of functioning can fluctuate over time. The comments illustrated the impact of this illness on the young people's lives:

'...I find it very difficult the negative [effects] CFS has had on my social side of life, with school and friends...'

\section{Theme 4: experiences with diagnosis and treatment}

Just over a fifth of the categories related to participants' experiences with treatment. Participants described contact with health professionals who lacked knowledge and understanding of CFS/ME and how to diagnose and treat it. Many of them were dismissive or disbelieving of participants' symptoms. One participant commented: 'Doctors do not seem to believe such an illness exists'.

Related to this, one participant commented on the need for more training for healthcare professionals in this area:

I must mention that I feel that there is not enough of the right sort of help and support being provided medically. It would be helpful if medical professionals were trained and educated on CFS/ME, especially on how each individual is affected differently by ME...

In addition, the following comment from a participant demonstrates that there can often be a lack of collaboration between different health professionals involved in the same patient's care:

[I would like]...a greater link between people in the medical profession, so they are equipped to deal with both ME and mental illnesses people get as a side effect of ME (OCD, Anxiety, Depression, Eating Disorders, etc.)...I have felt I've had to take advice from my ME OT and my mental health specialist and tried to find some sort of balance. This shouldn't be something I have to sort out, on top of everything else... 
Table 6 Table illustrating inductive content analysis of open-ended comments, including frequency of occurrence of each category and overarching themes

\begin{tabular}{|c|c|c|}
\hline Category & $\mathrm{n}$ & $\%$ \\
\hline Positive factors and sources of support & 40 & 27.6 \\
\hline Helpfulness of the charity & 13 & 9.0 \\
\hline Feeling connected to others with CFS/ME & 7 & 4.8 \\
\hline Receiving correspondence from the charity & 6 & 4.1 \\
\hline Charity acting as advocate for young people with CFS/ME & 3 & 2.1 \\
\hline Support from family & 2 & 1.4 \\
\hline Being able to access education & 2 & 1.4 \\
\hline Appreciating the small things in life & 1 & .7 \\
\hline Wanting more interaction with others with $\mathrm{CFS} / \mathrm{ME}$ as a source of support & 6 & 4.1 \\
\hline Experiences with diagnoses and treatment & 33 & 22.8 \\
\hline Dismissive or disbelieving health professionals & 6 & 4.1 \\
\hline Lack of knowledge of CFS/ME in healthcare professionals & 6 & 4.1 \\
\hline Given confusing advice or information & 5 & 3.4 \\
\hline Negative experiences from healthcare professionals & 4 & 2.8 \\
\hline Positive experiences of treatment & 3 & 2.1 \\
\hline Would like more information about treatments and how to access them & 3 & 2.1 \\
\hline Not enough medical help being provided & 2 & 1.4 \\
\hline Difficult to access help or treatment & 2 & 1.4 \\
\hline Lack of trust in medical profession & 1 & .7 \\
\hline Need for joined-up working by healthcare professionals & 1 & .7 \\
\hline The lived experience of having CFS/ME & 32 & 22.1 \\
\hline Impact of CFS/ME symptoms on everyday activities & 7 & 4.8 \\
\hline Fluctuating nature of CFS/ME symptoms & 6 & 4.1 \\
\hline Difficulties accessing education & 5 & 3.4 \\
\hline Comorbidity & 4 & 2.8 \\
\hline Negative impacts of CFS/ME & 3 & 2.1 \\
\hline $\mathrm{CFS} / \mathrm{ME}$ is more than just fatigue & 3 & 2.1 \\
\hline CFS/ME as part of identity & 2 & 1.4 \\
\hline Chronicity of fatigue & 1 & .7 \\
\hline Loss of independence & 1 & .7 \\
\hline Lack of awareness of CFS/ME and resulting stigma & 15 & 10.4 \\
\hline Lack of awareness of CFS/ME & 11 & 7.6 \\
\hline Negativity from other people with CFS/ME & 3 & 2.1 \\
\hline Stereotyping of CFS/ME & 1 & .7 \\
\hline Link between CFS/ME symptoms and other variables & 13 & 9 \\
\hline Factors linked with onset of CFS/ME symptoms & 7 & 4.8 \\
\hline External factors impacting on symptoms & 2 & 1.4 \\
\hline Low mood or anxiety as a consequence of CFS/ME symptoms & 2 & 1.4 \\
\hline Need for more research to increase understanding of CFS/ME & 2 & 1.4 \\
\hline Course and prognosis of CFS/ME & 12 & 8.3 \\
\hline Feeling better & 7 & 4.8 \\
\hline Comparison to life before CFS/ME & 2 & 1.4 \\
\hline CFS/ME does not last forever & 1 & .7 \\
\hline Gradual improvement & 1 & .7 \\
\hline Feeling hopeful & 1 & .7 \\
\hline
\end{tabular}

Over-arching themes are in shown in bold type. The total frequency of occurrences for all categories under one theme were summed to obtain a total $\mathrm{n}$ and percentage for each theme, which are shown in bold next to each category 


\section{Theme 5: course and prognosis of $\mathrm{CFS} / \mathrm{ME}$}

Within this theme, participants reported that they were feeling better, and there were comments suggesting that improvement had been slow and gradual. One participant commented: 'At the moment I am feeling slightly better, but it has taken a long time to get this far'.

\section{Theme 6: lack of awareness of CFS/ME by others and resulting stigma}

Just over $10 \%$ of the categories were related to lack of awareness of CFS/ME by others, and the comments from participants suggested that there is a lack of awareness of CFS/ME both in the medical profession (as mentioned in theme 4: Experiences with diagnosis and treatment) and in the wider community. One participant commented: 'I think more people need to know about this condition, as when my mum says to people I have ME and chronic pain syndrome, no [one] understands it, they think it's a cold or yuppy flu!'.

\section{Discussion}

The aim of this study was to explore the experiences of young people with severe CFS/ME and to investigate factors associated with fatigue, social adjustment and work/ school attendance. Participants' responses to the questionnaire demonstrated the profound disability associated with having severe CFS/ME: almost three quarters of participants reported using assistive equipment, and more than two-thirds reported using a wheelchair. Only around a quarter of patients had been able to attend school, college, or work in the past year. The findings support previous research which shows the negative impacts that symptoms of CFS/ME can have on a young person's life. Symptoms of CFS may mean that individuals with CFS have to miss large amounts of school, or give up valued and enjoyable activities, a consequence of which may be social isolation (Crawley \& Sterne, 2009; Jelbert et al., 2010; Parslow et al., 2017).

Many participants in this study reported a lack of awareness and understanding of CFS/ME by their peers, schools, healthcare professionals, and in the wider community. Participants described the stigma of having an illness that was misunderstood by others. This led to difficulties such as problems with accessing education, despite this being deeply meaningful and valuable to them. Many participants also reported barriers in obtaining a diagnosis of CFS/ME and accessing treatment for it. Healthcare professionals' lack of understanding and awareness of $\mathrm{CFS} / \mathrm{ME}$ and medical uncertainty about this illness has been reported previously (Bowen, et al., 2005; Jelbert et al., 2010; Webb et al., 2011). It is clear that there is still work to be done in raising awareness about CFS/ME in the health and education sectors and within the general public, in order to reduce some of the stigma associated with symptoms and to help people with severe CFS/ME to feel less isolated.

Although this was a naturalistic study and not an evaluation of treatment, it is notable that the majority of participants reported that they had accessed some form of treatment, and yet the mean scores for fatigue and social functioning had not changed considerably by T2. Another interesting finding was that access to treatment was associated with lower work/school attendance at the univariable level, but the variable itself was not a significant predictor when included with other variables within a multivariable model. It could be that participants who accessed treatment required more time away from school/work in order to attend appointments. However, it may also be that the effects of treatment would not be seen within such a short period of time.

The finding that fear avoidance beliefs were associated with worse social adjustment supplements earlier findings showing that illness-related beliefs and cognitions may be associated with poorer functional impairment (Richards et al., 2005) in young people with CFS/ME. This suggests that cognitions such as fear avoidance beliefs may be an important factor to consider in the understanding of the processes that maintain fatigue and disability in CFS/ME. This study did not allow for causal inferences to be made, but an alternative explanation of the findings is that fearful beliefs developed as a result of being so severely ill. Either way they could be targeted as part of a cognitive-behavioural treatment package.

Another key finding was that almost a third of participants in this study scored above the cut-off threshold for the presence of depression. This is comparable with the rates of comorbid depression (29-34.6\%) reported in previous clinical cohort studies of adolescents with CFS/ME (Bould et al., 2013; Loades et al., 2017) and adds to research showing that rates of depression are generally higher in adolescents with CFS than those in the general population (Bould et al., 2013). Similarly, the rates of anxiety reported by children and adolescents with CFS/ ME were higher than those reported by children and adolescents in the general population (Stoll et al., 2017; Crawley et al., 2009). This finding also supports existing research in which young people with symptoms of CFS/ ME report negative impacts of symptoms such as reduction in activity, school absence and difficulties maintaining social relationships. This is in addition to the negative emotional impact of CFS/ME and difficult feelings such as loss, isolation, and loneliness that are often reported by 
young people with CFS/ME (Jelbert et al., 2010; Parslow et al., 2017; Taylor et al., 2016). Previous research also suggests that having an illness like CFS/ME is associated with low mood and depression as well as anxiety, but the direction of causality is unclear (Bould et al., 2013; Taylor et al., 2016). There is a lack of evidence about which treatments are effective for young people with CFS/ME who also have comorbid depression, and further research is needed in this area (Loades et al., 2016).

Fear avoidance beliefs, which were found to be an important factor associated with social adjustment in this study, can be targeted using rehabilitative treatments such as CBT. There is growing evidence that this type of treatment is effective for the treatment of CFS/ME. CBT has been shown to be effective for the treatment of CFS/ ME in adolescents and young people, in terms of reduced fatigue and increased school attendance (Chalder et al., 2010; Nijhof et al., 2012; Stulemeijer et al., 2005). Followup studies have shown that the positive effects of CBT can be maintained long term (Knoop et al., 2008; Lloyd et al., 2012).

However there is a dearth of research about treatment options for young people with severe CFS/ME. One option for individuals who are too unwell to attend hospital appointments is home-based rehabilitative treatment. Burgess and Chalder reported that an adolescent with severe $\mathrm{CFS} / \mathrm{ME}$ who received treatment using this approach made a full recovery, returning to school and showing reduced fatigue and normal functioning (Burgess \& Chalder, 2011). This was despite the fact that the adolescent had been bedbound. The approach consisted of stabilising activity levels and addressing fearful beliefs about activity. The authors suggested that a strong therapeutic relationship with the patient and family was key to the treatment's positive outcome. Following on from this promising case report, a case-series found that a small group of adolescents $(n=6)$ who received home-based family-focused CBT recovered, and that gains were maintained at follow-up (Burgess et al., 2018). Of course further research on treatment effectiveness is needed, in a controlled setting and on a larger scale.

There are some biological characteristics which were not measured in this study but may impact on fatigue and levels of functioning. For example, sleep problems are commonly reported in chronic fatigue syndrome (Gotts et al., 2014). Sleep quality appears to be poorer in adolescents with chronic fatigue syndrome compared to healthy controls (Josev et al., 2017; Snodgrass et al., 2015). A recent birth cohort study found that participants who developed chronic disabling fatigue during adolescence had a shorter sleep duration during their early childhood. The authors suggested that sleep abnormalities may be a risk factor for the development of CFS/ME, and that this may be associated with dysfunction of an underlying physiological mechanism (Collin et al., 2018b). Sleep problems may also be associated with disruptions to circadian rhythms, although research on this is inconsistent and further higher quality research is needed (Snodgrass et al., 2015). Within the confines of this study, it was not possible to undertake measurements of anthropometric characteristics such as weight. This may be an important factor to consider, because there is some evidence that high levels of obesity are associated with high levels of fatigue (Lim et al., 2008). However there is an absence of research on the link between fatigue and obesity in adolescents and young people.

To our knowledge, this is the first study to explore the experiences of young people with severe CFS/ME. However, the study has several limitations which should be taken into account. Despite the initial attempt to recruit over 300 participants this small sample of 51 consisted of members of a charity for young people with CFS/ME. Therefore the sample may not be representative of young people with severe CFS/ME within the general population. It follows then that the findings of this study may not be generalizable to other settings and populations. Moreover, there was a gender imbalance (the majority of participants were female). Another limitation is the fact that participants self-reported their diagnosis which we were not able to verify. This means that we cannot be sure that they met all the requirements of the Oxford criteria. Neither can we say with certainty that the diagnosis had been verified by a specialist.

There was no control group in the current study. This limits the interpretation of the findings, and means that causal inferences cannot be made. It may be useful to compare people with CFS to an illness control group in order to ascertain whether factors (such as fear avoidance beliefs) are also associated with fatigue or functioning in other illnesses.

Due to the wide age-range of participants included in this study, there may have been differences between the younger and older participants in this group. The brain continues to undergo changes as it matures throughout adolescence and early adulthood. Development of some of the important fronto-temporal connections in the brain continues until at least the age of 25 (Lebel et al., 2008). However, these differences could not be investigated due to lack of adequate power. It was not possible to investigate any causal associations between the variables measured in this study due to the short follow-up period. Given these caveats, the findings of the current study should be interpreted with caution.

Future research could involve longitudinal studies including a larger sample with a longer follow-up period. It could also be possible to compare groups of children, adolescents, and young adults, in order to investigate whether these age groups show differences in terms of the factors that are associated with outcome. 
In summary, this study found that severe CFS/ME negatively impacted on many aspects of young people's lives, and that fearful beliefs were associated with poorer social adjustment. There is a need for further research about how severe CFS in young people can be effectively treated.

Acknowledgements We are extremely grateful to the young people and their families who took part in the study and helped to pilot the questionnaire. We thank the team at the organisation Association of Young People With M.E., for recruiting participants and collecting data.

Funding TC acknowledges the financial support of the Department of Health via the National Institute for Health Research (NIHR) Specialist Biomedical Research Centre for Mental Health award to the South London and Maudsley NHS Foundation Trust (SLaM) and the Institute of Psychiatry at King's College London. The views expressed in this article are those of the authors and not necessarily those of the NHS, the NIHR or the Department of Health.

\section{Compliance with Ethical Standards}

Conflicts of interest Trudie Chalder received ad hoc payments for conducting workshops on evidence-based treatments for persistent physical symptoms; grants from NIHR programme grants, HTA, RfPB, Guy's and St Thomas Charity, King's Challenge Fund, Muscular Dystrophy, Multiple Sclerosis Society. Personal financial interests: Trudie Chalder is the author of several self-help books on chronic fatigue and received royalties in the past. She received expenses and ad hoc payment for role as external examiner NUI Galway and Waterford Institute of Technology. She received expenses for keynote speeches at UK Society for Behavioural Medicine, BABCP Conferences (travel and accommodation). Mary Burgess receives royalties for the self-help book Overcoming chronic fatigue. Sheila Ali and Lucy Adamczyk declare that they have no conflict of interest.

Human and animal rights and Informed Consent All procedures followed were in accordance with ethical standards of the responsible committee on human experimentation (institutional and national) and with the Helsinki Declaration of 1975, as revised in 2000. The study received ethical approval from the Psychiatry, Nursing and Midwifery sub-committee of King's College London Research Ethics Committee. Informed consent was obtained from all patients for being included in the study.

Open Access This article is distributed under the terms of the Creative Commons Attribution 4.0 International License (http:// creativecommons.org/licenses/by/4.0/), which permits unrestricted use, distribution, and reproduction in any medium, provided you give appropriate credit to the original author(s) and the source, provide a link to the Creative Commons license, and indicate if changes were made.

\section{References}

Arita, J. H., Lin, J., Pinho, R. S., Minett, T. S., de Souza Vitalle, M. S., Fisberg, M., et al. (2013). Adolescents with chronic migraine commonly exhibit depressive symptoms. Acta Neurologica Belgica, 113, 61-65.
Arroll, B. (2003). Screening for depression in primary care with two verbally asked questions: Cross sectional study. British Medical Journal, 327, 1144-1146.

Bakken, I. J., Tveito, K., Aaberg, K. M., Ghaderi, S., Gunnes, N., Trogstad, L., et al. (2016). Comorbidities treated in primary care in children with chronic fatigue syndrome/myalgic encephalomyelitis: A nationwide registry linkage study from Norway. BMC Family Practice, 17, 128.

Bould, H., Collin, S. M., Lewis, G., Rimes, K., \& Crawley, E. (2013). Depression in paediatric chronic fatigue syndrome. Archives of Disease in Childhood, 98, 425-428.

Bowen, J., Pheby, D., Charlett, A., \& McNulty, C. (2005). Chronic fatigue syndrome: A survey of GPs' attitudes and knowledge. Family Practice An International Journal, 22, 389-393.

Brurberg, K. G., Fonhus, M. S., Larun, L., Flottorp, S., \& Malterud, K. (2014). Case definitions for chronic fatigue syndrome/ myalgic encephalomyelitis(CFS/ME): A systematic review. British Medical Journal Open, 4, e003973. https://doi.org/10. 1136/bmjopen-2013-003973

Burgess, M., \& Chalder, T. (2011). Adolescents with severe chronic fatigue syndrome can make a full recovery. BMJ Case Reports. https://doi.org/10.1136/bcr.01.2011.3716

Burgess, M., Lievesley, K., Ali, S., \& Chalder, T. (2018). Homebased family-focused rehabilitation for adolescents with severe chronic fatigue syndrome: Recovery is possible. Clinical Child Psychology and Psychiatry. Advance online publication. https:// doi.org/10.1177/1359104518794764

Cella, M., \& Chalder, T. (2010). Measuring fatigue in clinical and community settings. Journal of Psychosomatic Research, 69, $17-22$.

Cella, M., Sharpe, M., \& Chalder, T. (2011). Measuring disability in patients with chronic fatigue syndrome: reliability and validity of the work and social adjustment scale. Journal of Psychosomatic Research, 71, 124-128.

Chalder, T., Berelowitz, G., Pawlikowska, T., Watts, L., Wessely, S., Wright, D., et al. (1993). Development of a fatigue scale. Journal of Psychosomatic Research, 37, 147-153.

Chalder, T., Deary, V., Husain, K., \& Walwyn, R. (2010). Family focused cognitive behaviour therapy versus psycho-education for chronic fatigue syndrome in 11-18 year olds: A randomized controlled treatment trial. Psychological Medicine, 40, $1269-1279$.

Chalder, T., Goodman, R., Wessely, S., Hotopf, M., \& Meltzer, H. (2003). Epidemiology of chronic fatigue syndrome and self reported myalgic encephalomyelitis in 5-15 year olds: Cross sectional study. British Medical Journal, 327, 654-655.

Collin, S. M., Norris, T., Deere, K. C., Jago, R., Ness, A. R., \& Crawley, E. (2018a). Physical activity at age 11 years and chronic disabling fatigue at ages 13 and 16 years in a UK birth cohort. Archives of Disease in Childhood, 103, 586-591.

Collin, S. M., Norris, T., Gringras, P., Blair, P. S., Tilling, K., \& Crawley, E. (2018b). Childhood sleep and adolescent chronic fatigue syndrome (CFS/ME): Evidence of associations in a UK birth cohort. Sleep Medicine, 46, 26-36.

Collin, S. M., Norris, T., Nuevo, R., Tilling, K., Joinson, C., Sterne, J. A., et al. (2016). Chronic fatigue syndrome at age 16 years. Pediatrics, 137, e20153434.

Crawley, E., Emond, A. M., \& Sterne, J. A. (2011). Unidentified chronic fatigue syndrome/myalgic encephalomyelitis (CFS/ME) is a major cause of school absence: Surveillance outcomes from school-based clinics. British Medical Journal Open, 1, e000252.

Crawley, E., Hunt, L., \& Stallard, P. (2009). Anxiety in children with CFS/ME. European Child and Adolescent Psychiatry, 18, 683-689.

Crawley, E., \& Sterne, J. A. (2009). Association between school absence and physical function in paediatric chronic fatigue 
syndrome/myalgic encephalopathy. Archives of Disease in Childhood, 94, 752-756.

Farmer, A., Fowler, T., Scourfield, J., \& Thapar, A. (2004). Prevalence of chronic disabling fatigue in children and adolescents. British Journal of Psychiatry, 184, 477-481.

Fukuda, K., Straus, S. E., Hickie, I., Sharpe, M. C., Dobbins, J. G., \& Komaroff, A. (1994). The chronic fatigue syndrome: A comprehensive approach to its definition and study. International Chronic Fatigue Syndrome Study Group. Annals of Internal Medicine, 121, 953-959.

Gill, A. C., Dosen, A., \& Ziegler, J. B. (2004). Chronic fatigue syndrome in adolescents: A follow-up study. Archives of Pediatrics and Adolescent Medicine, 158, 225-229.

Gotts, Z. M., Ellis, J. G., Newton, J. L., \& Deary, V. (2014). The role of sleep in chronic fatigue syndrome: A narrative review. Fatigue: Biomedicine Health \& Behavior, 2, 163-184.

Gray, S. E., \& Rutter, D. R. (2007). Illness representations in young people with Chronic Fatigue Syndrome. Psychology \& Health, 22, 159-174.

Hickie, I., Kirk, K., \& Martin, N. (1999). Unique genetic and environmental determinants of prolonged fatigue: A twin study. Psychological Medicine, 29, 259-268.

Hsieh, H.-F., \& Shannon, S. E. (2005). Three approaches to qualitative content analysis. Qualitative Health Research, 15, $1277-1288$.

Jelbert, R., Stedmon, J., \& Stephens, A. (2010). A qualitative exploration of adolescents' experiences of chronic fatigue syndrome. Clinical Child Psychology and Psychiatry, 15, 267-283.

Josev, E. K., Jackson, M. L., Bei, B., Trinder, J., Harvey, A., Clarke, C., et al. (2017). Sleep quality in adolescents with chronic fatigue syndrome/myalgic encephalomyelitis(CFS/ME). Journal of Clinical Sleep Medicine, 13, 1057-1066.

IBM Corp. (2015). IBM SPSS Statistics for Windows, Version 23.0. [Computer software]. Armonk, NY: IBM Corp.

Katz, B. Z., Shiraishi, Y., Mears, C. J., Binns, H. J., \& Taylor, R. (2009). Chronic fatigue syndrome after infectious mononucleosis in adolescents. Pediatrics, 124, 189-193.

Knight, S., Harvey, A., Lubitz, L., Rowe, K., Reveley, C., Veit, F., et al. (2013). Paediatric chronic fatigue syndrome: Complex presentations and protracted time to diagnosis. Journal of Paediatrics and Child Health, 49, 919-924.

Knoop, H., Stulemeijer, M., de Jong, L. W., Fiselier, T. J., \& Bleijenberg, G. (2008). Efficacy of cognitive behavioral therapy for adolescents with chronic fatigue syndrome: Long-term follow-up of a randomized, controlled trial. Pediatrics, 121, e619-e625.

Kroenke, K., Spitzer, R. L., Williams, J. B., Monahan, P. O., \& Lowe, B. (2007). Anxiety disorders in primary care: Prevalence, impairment, comorbidity, and detection. Annals of Internal Medicine, 146, 317-325.

Lebel, C., Walker, L., Leemans, A., Phillips, L., \& Beaulieu, C. (2008). Microstructural maturation of the human brain from childhood to adulthood. NeuroImage, 40, 1044-1055.

Lievesley, K., Rimes, K. A., \& Chalder, T. (2014). A review of the predisposing, precipitating and perpetuating factors in chronic fatigue syndrome in children and adolescents. Clinical Psychology Review, 34, 233-248.

Lim, W., Thomas, K. S., Bardwell, W. A., \& Dimsdale, J. E. (2008). Which measures of obesity are related to depressive symptoms and in whom? Psychosomatics, 49, 23-28.

Lloyd, S., Chalder, T., \& Rimes, K. (2012). Family-focused cognitive behaviour therapy versus psycho-education for adolescents with chronic fatigue syndrome. Long-term follow-up of an RCT. Behaviour Research and Therapy, 50, 719-725.
Loades, M. E., Rimes, K. A., Ali, S. A., Lievesley, K., \& Chalder, T. (2017). The presence of co-morbid mental health problems in a cohort of adolescents with chronic fatigue syndrome. Clinical Child Psychology and Psychiatry. Advance online publication. https://doi.org/10.1177/1359104517736357

Loades, M. E., Sheils, E. A., \& Crawley, E. (2016). Treatment for paediatric chronic fatigue syndrome or myalgic encephalomyelitis (CFS/ME) and comorbid depression: A systematic review. British Medical Journal Open, 6, e012271.

Loades, M.W., Rimes, K., Ali S., Lievesley K., \& Chalder T. (in submission). Cognitive and behavioural responses to symptoms in adolescents with Chronic Fatigue Syndrome (CFS): A Case Control Study nested within a cohort.

Moss, J. (2005). Development of a functional ability scale for children and young people with myalgic encephalopathy (ME)/chronic fatigue syndrome (CFS). Journal of Child Health Care, 9, 20-30.

Mundt, J. C., Marks, I. M., Shear, M. K., \& Greist, J. H. (2002). The work and social adjustment scale: A simple measure of impairment in functioning. British Journal of Psychiatry, 180, 461-464.

Nijhof, S. L., Bleijenberg, G., Uiterwaal, C. S., Kimpen, J. L., \& van de Putte, E. M. (2012). Effectiveness of internet-based cognitive behavioural treatment for adolescents with chronic fatigue syndrome (FITNET): A randomised controlled trial. Lancet, 379, 1412-1418.

Norris, T., Collin, S. M., Tilling, K., Nuevo, R., Stansfeld, S. A., Sterne, J. A., et al. (2017). Natural course of chronic fatigue syndrome/myalgic encephalomyelitis in adolescents. Archives of Disease in Childhood, 102, 522-528.

Parslow, R. M., Harris, S., Broughton, J., Alattas, A., Crawley, E., Haywood, K., et al. (2017). Children's experiences of chronic fatigue syndrome/myalgic encephalomyelitis (CFS/ME): A systematic review and meta-ethnography of qualitative studies. British Medical Journal Open, 7, e012633.

Rangel, L., Garralda, M. E., Levin, M., \& Roberts, H. (2000). The course of severe chronic fatigue syndrome in childhood. Journal of the Royal Society of Medicine, 93, 129-134.

Richards, J. (2000). Chronic fatigue syndrome in children and adolescents: A review article. Clinical Child Psychology and Psychiatry, 5, 31-51.

Richards, J., Turk, J., \& White, S. (2005). Children and adolescents with Chronic Fatigue Syndrome in non-specialist settings: Beliefs, functional impairment and psychiatric disturbance. European Child and Adolescent Psychiatry, 14, 310-318.

Rimes, K. A., Goodman, R., Hotopf, M., Wessely, S., Meltzer, H., \& Chalder, T. (2007). Incidence, prognosis, and risk factors for fatigue and chronic fatigue syndrome in adolescents: A prospective community study. Pediatrics, 119, e603-e609.

Ryan, E. G., Vitoratou, V., Goldsmith, K. A., \& Chalder, T. (2017). Psychometric properties and factor structure of a shortened version of the cognitive behavioural responses questionnaire (CBRQ). Psychosomatic Medicine, 80, 230-237.

Sankey, A., Hill, C. M., Brown, J., Quinn, L., \& Fletcher, A. (2006). A follow-up study of chronic fatigue syndrome in children and adolescents: Symptom persistence and school absenteeism. Clinical Child Psychology and Psychiatry, 11, 126-138.

Sharpe, M., Archard, L. C., Banatvala, J. E., Borysiewicz, L. K., Clare, A. W., David, A., et al. (1991). A report-chronic fatigue syndrome: Guidelines for research. Journal of the Royal Society of Medicine, 84, 118-121.

Skerrett, T. N., \& Moss-Morris, R. (2006). Fatigue and social impairment in multiple sclerosis: The role of patients' cognitive and behavioral responses to their symptoms. Journal of Psychosomatic Research, 61, 587-593. 
Snodgrass, K., Harvey, A., Scheinberg, A., \& Knight, S. (2015). Sleep disturbances in pediatric chronic fatigue syndrome: A review of current research. Journal of Clinical Sleep Medicine, 11, $757-764$

Sommer, B. (2006). Content analysis. [Course notes] Retrieved from http://psc.dss.ucdavis.edu/sommerb/sommerdemo/

Stahl, D., Rimes, K. A., \& Chalder, T. (2014). Mechanisms of change underlying the efficacy of cognitive behaviour therapy for chronic fatigue syndrome in a specialist clinic: A mediation analysis. Psychological Medicine, 44, 1331-1344.

Stoll, S. V. E., Crawley, E., Richards, V., Lal, N., Brigden, A., \& Loades, M. E. (2017). What treatments work for anxiety in children with chronic fatigue syndrome/myalgic encephalomyelitis(CFS/ME)? Systematic review. BMJ Open, 7, e015481. https://doi.org/10.1136/bmjopen-2016-015481

Strassheim, V., Lambson, R., Hackett, K. L., \& Newton, J. L. (2017). What is known about severe and very severe chronic fatigue syndrome? A scoping review. Fatigue: Biomedicine Health \& Behavior, 5, 167-183.

Stulemeijer, M., de Jong, L. W., Fiselier, T. J., Hoogveld, S. W., \& Bleijenberg, G. (2005). Cognitive behaviour therapy for adoles- cents with chronic fatigue syndrome: Randomised controlled trial. British Medical Journal, 330, 14.

Taylor, A. K., Loades, M., Brigden, A. L. C., Collin, S. M., \& Crawley, E. (2016). 'It's personal to me': A qualitative study of depression in young people with CFS/ME. Clinical Child Psychology and Psychiatry, 22, 326-340.

The National Task Force on Chronic Fatigue Syndrome. Report from the National Task Force on chronic fatigue syndrome (CFS), post viral syndrome (PVFS), myalgic encephalomyelitis (ME). Bristol: Westcare, The National Task Force on Chronic Fatigue Syndrome, 1994.

Webb, C. M., Collin, S. M., Deave, T., Haig-Ferguson, A., Spatz, A., \& Crawley, E. (2011). What stops children with a chronic illness accessing health care: A mixed methods study in children with chronic fatigue syndrome/myalgic encephalomyelitis (CFS/ME). BMC Health Services Research, 11, 308.

Publisher's Note Springer Nature remains neutral with regard to jurisdictional claims in published maps and institutional affiliations. 